\title{
Análisis Morfológico de Patentes para Desarrollar un Producto de Seguridad Vehicular
}

\author{
Morphologic Patent Analysis as to Develop a Vehicular \\ Security Product
}

\author{
Sara Ortiz Cantúl, Álvaro Pedroza Zapata², Esteban Martinez Guerrero ${ }^{3}$
}

\begin{abstract}
En este trabajo se proporcionan las bases de inteligencia tecnológica para un proyecto de seguridad vehicular, adoptando el modelo de innovación propuesto por Myers y Marquis, Se hizo un análisis morfológico de patentes basado en palabras clave para buscar las configuraciones ya utilizadas (protegidas) y se identifican las configuraciones aún no empleadas para identificar las oportunidades tecnológicas a desarrollar. Este análisis se presta para: I) conocer el estado del arte y no comenzar de cero o inventar el hilo negro, 2) evitar invadir propiedad industrial de terceros, y 3) descubrir huecos de oportunidades tecnológicas.
\end{abstract}

Keywords: inteligencia tecnológica; detección de necesidades; análisis morfológico de patentes.

This paper provides the basis for technological intelligence for a vehicle safety project, specifically to avoid rear-end collisions between cars. The framework adopts the general model of innovation proposed by Myers and Marquis. A morphological analysis of patents based on keywords was done to find the settings already used (protected) in the patents analyzed. By listing the occupied configurations of collected patents, the unoccupied territory of configurations are suggested as technology opportunities. This analysis provides: I) the state of art and not to reinvent the wheel, 2) avoid infringement to industrial property, and 3) discover white spaces as technological opportunities.

Keywords: technology intelligence; identifying needs; morphological analysis of patents.

\footnotetext{
${ }^{1.3}$ Departamento de Electrónica, Sistemas e Informática, ITESO, Periférico Sur Manuel Gómez Morín \#8585, Tlaquepaque, Jalisco, México, CP 45604. Phone: 36693434. 'Anexo: 3 I 50. E-mail: sortiz@iteso.mx 3Anexo: 3177. E-mail: margres@iteso.mx

²Departamento de Administración, Finanzas y Mercadología, ITESO, Periférico Sur Manuel Gómez Morín \#8585, Tlaquepaque, Jalisco, México, CP 45604. Phone: 36693434. Anexo: 3429. E-mail: apedroza@iteso.mx
}

ISSN: 07 I8-2724. (http://www.jotmi.org)

Journal of Technology Management \& Innovation (c) Universidad Alberto Hurtado, Facultad de Economía y Negocios. 


\section{Antecedentes Teóricos}

La innovación ocurre cuando las necesidades se juntan a las tecnologías que tratan esas necesidades y el proceso nuevo resultante, el producto y las ideas del servicio se desarrollan de una manera responsable que balancea los riesgos y las recompensas de hacer algo nuevo y comienza con la colección de información a cerca de:

- Metas y capacidades de la empresa.

- Las metas actuales y las necesidades no explícitas de sus clientes.

- Las tecnologías potencialmente disponibles para satisfacer esas necesidades.

- Las capacidades y las intenciones de sus competidores actuales y potenciales.

El concepto básico es simple: la innovación ocurre cuando hay un empate de una necesidad nueva o emergente con una tecnología existente o emergente, $y$ las empresas industriales seleccionan y desarrollan las mejores ideas usando un proceso dirigido que balancee los riesgos y las variables desconocidas. En la Figura I se adapta del modelo de la innovación de Marquis y Myers.

El proceso aquí planteado es para centrarse en el "extremo delantero borroso" y parte de la experiencia y la investigación necesarias para la innovación y la toma de decisiones de la organización con el objeto de proporcionar un acercamiento basado en la relación de las necesidades de clientes/mercados y las tecnologías de una manera estructurada (Pedroza y Ortiz, 2008).

El proceso de innovación comienza con recabar el conocimiento de las necesidades del consumidor por una parte, $y$ de la oportunidades tecnológicas por otra. El indagar sobre la demanda presupone la disponibilidad de datos provenientes del mercado, mientras que los aspectos técnicos recurren a información tecnocientífica. En otras palabras, durante esta fase se debe enterar de los productos que son demanda- dos por el consumidor, pudiendo ser una demanda actual o potencial. Se debe cuestionar sobre qué cosas se necesitan y/o cómo mejorar los productos ya existentes. También se debe obtener conocimiento del estado del arte en materia tecnológica, ya que se partirá de estas bases para estimar la factibilidad técnica.

Esta etapa es especialmente importante pues el hecho de que exista demanda no implica que haya una solución técnica apropiada para satisfacerla, $y$ viceversa, el tener suficiente conocimiento técnico no garantiza que un producto tenga aceptación de mercado.

Para generar y proponer ideas de innovación es necesario fusionar creativamente el conocimiento obtenido sobre la demanda con el de la factibilidad técnica. Al correlacionar de una manera diferente lo que se necesita con lo que se puede hacer de acuerdo a los recursos disponibles da como resultado la idea o el diseño de la innovación. Este proceso está altamente influenciado por la calidad y la cantidad de la información técnica y de mercado que esté a disposición del creativo, y más importante aún, por la asociación que la persona haga de estos dos elementos. Obviamente, es muy difícil que una sola persona se dedique a recabar la información y posteriormente, que entienda todos los aspectos técnicos, sociales y económicos envueltos en el proceso. Lo más común es que se tenga un equipo de trabajo acompañado de un esfuerzo cultural a lo largo de la organización a través de comunidades de innovación.

La idea es el punto de partida para identificar las necesidades a resolver mediante alguna solución tecnológica, la cual se materializa en una hoja de concepto donde se defina lo siguiente: I) la funcionalidad del producto a desarrollar, 2) las necesidades básicas a satisfacer, 3 ) el mercado meta al que va dirigido el producto, 4) los hábitos de consumo actuales, 5)las variables competitivas, 6)las características funcionales del producto, 7) los ángulos de diferenciación frente a los productos similares que existen en el mercado, 8) los productos en competencia y el estado actual de la tecnología de

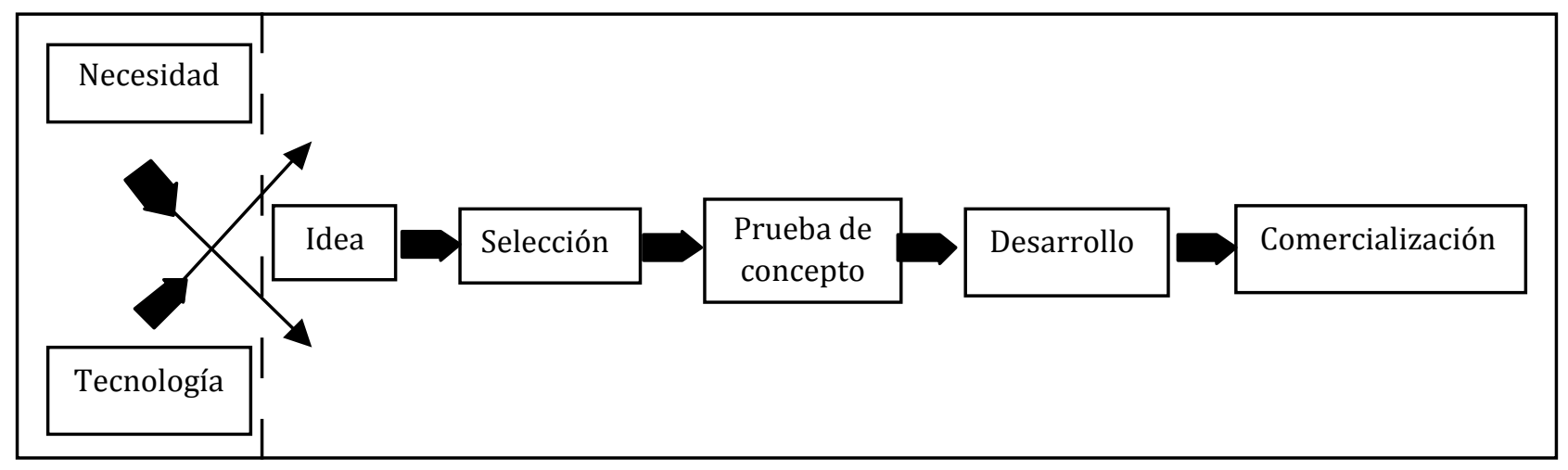

Figura I. Modelo de innovación (adaptado de Marquis y Myers)

ISSN: 07 I8-2724. (http://www.jotmi.org)

Journal of Technology Management \& Innovation (c) Universidad Alberto Hurtado, Facultad de Economía y Negocios. 
los productos en competencia, 9) ciclo de vida del producto o de la industria, 10) el período de tiempo proyectado para el producto, II) aspectos tecnológicos del desarrollo, 12) restricciones para la el desarrollo del producto y 13) productos complementarios a añadir al producto principal.

Ya que se ha planteado la hipótesis o el concepto del diseño, se tiene que decidir si conviene o no introducir la innovación; para ello se consideran factores como: la probabilidad de éxito técnico, el costo de desarrollo y fabricación, el tiempo necesario para llevar a cabo el desarrollo, la probabilidad de éxito en las actividades de comercialización y la rentabilidad del proyecto, entre otros.

El tener lista una idea o un diseño es sólo la presentación de un problema que tiene que ser resuelto siempre y cuando valga la pena su consideración en términos económicos, de acuerdo al paso anterior.

La inteligencia tecnológica implica la búsqueda de información que generalmente se encuentra disponible en bancos de datos computarizados, o bien, a través de técnicos expertos en el área. La finalidad de esta búsqueda es contar con información más profunda sobre el estado del arte, o sea, los últimos avances en materia tecnológica.

Cuando hace falta más información que ya no se encuentra en forma de literatura, es necesario emprender actividades de investigación y desarrollo, que arrojen más luz sobre el problema con el objeto de detectar nuevas oportunidades, para retomar viejos proyectos, o bien, para descartar proyectos actuales. Como resultado, se genera una solución prototipo; como su nombre lo indica, esta es una solución que todavía necesita refinarse, porque pueden quedar algunas interrogantes sobre su aplicación.

Para su refinación se hecha mano de la información técnica investigada en la etapa anterior, junto con los resultados de los programas de investigación y desarrollo. La combinación de esta información puede concretar en un invento, susceptible a patentarse.

La etapa de desarrollo implica escalar la solución prototipo a un nivel de producción, para ello, se hace necesaria la construcción de plantas piloto para hacer pruebas de verificación del funcionamiento de la solución. También incluye una verificación detallada de la demanda del mercado.

Finalmente, en la última etapa hace alusión al arranque del proceso de manufactura. Así, la innovación empieza a expandirse hacia otras organizaciones. Ya no se puede hablar de un riesgo técnico, sino más bien de un riesgo económico, puesto que aquí es cuando se incurre en los mayores costos.

La importancia que tiene este modelo de innovación es que puede ayudar al empresario a entender que las ideas, para

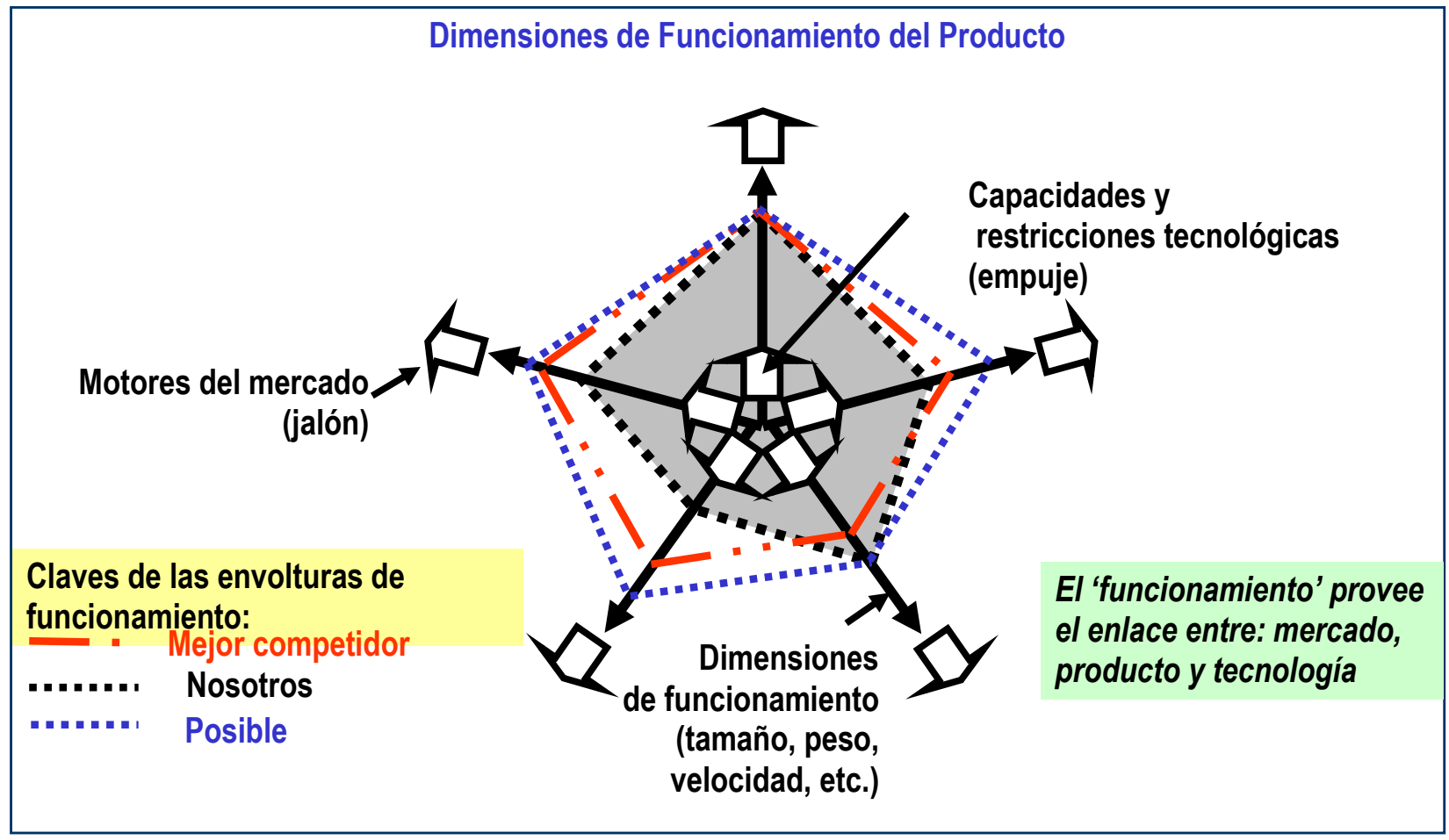

Figura 2. Dimensiones de Funcionamiento del Producto

ISSN: 07 I8-2724. (http://www.jotmi.org)

Journal of Technology Management \& Innovation (C) Universidad Alberto Hurtado, Facultad de Economía y Negocios. 
convertirse en innovaciones, deben tener una fundamentación tecnológica y un respaldo de mercado; además deben contar con el empuje de un emprendedor. Por lo tanto, un buen empresario debe conjuntar un equipo de personas que aporten los diferentes conocimientos y habilidades mencionadas.

\section{Necesidades de Clientes y Mercados}

Como parte de la planeación es necesario conocer las necesidades del cliente, explícitas y no explícitas. La responsabilidad de identificar las necesidades del cliente se encuentra compartida entre mercadotecnia y tecnología, sin embargo la responsabilidad de asegurar que exista el conocimiento y la implementación de los quehaceres para adquirir dicha plataforma de conocimiento es enteramente responsabilidad de tecnología.

Además de tener bien identificadas las necesidades y valores del cliente y las ventajas por obtener, dos cosas importantes a conocer para poder tomar un rumbo tecnológico definido:

- Dónde se encuentra los principales competidores

- Cuál será el alcance de los proyectos tecnológicos

El primer punto se refiere principalmente a la competencia y el segundo punto al alcance que se debe dar a los proyectos tecnológicos en el sentido de recursos y de los límites necesarios para poder efectuar una buena propuesta; es un "conocimiento interno".

La vigilancia tecnológica (VT) del principal competidor así como la definición y alcance de los proyectos necesarios implican una gran cantidad de información que requiere ser administrada para su análisis y unificada para su síntesis. La síntesis de laVT se lleva a cabo mediante un "Mapa Tecnológico" en el que se engloban la posición actual de la empresa, la de los competidores, el límite científico y los proyectos tecnológicos necesarios para alcanzar la posición deseada. Este mapa efectúa un proceso de síntesis de la VT, misma que da un rumbo organizado a los proyectos tecnológicos. Esta información abundante pero sintetizada, es suficiente para llevar a cabo una discusión de posibilidades técnicas de manera organizada permitiendo así observar tanto el panorama general como el detalle técnico (Ver Figura 2).

\section{Análisis Morfológico Convencional}

El análisis morfológico (AM) es una técnica combinatoria de ideación creativa, la cual se realiza descomponiendo un problema, sistema o concepto en sus elementos esenciales. El punto clave del análisis morfológico es la generación de una matriz, la cual se construye a través de los rasgos o atributos del problema, sistema o concepto en cuestión. El objetivo de la construcción de la matriz es multiplicar las relaciones entre tales partes dando como resultado combinaciones que quizás al inicio del análisis no se habían planteado.

La idea básica del AM es que el objeto problema es descompuesto en varias dimensiones a través de las cuales puede describirse de una manera comprensiva y detallada (Wissema, 1976). De manera simple, un sistema está compuesto de un número de subsistemas, cada uno de los cuales puede ser conformado de diferentes maneras. El AM identifica las diferentes formas que puede tomar cada dimensión y, combinando estas, examina todas las posibles alternativas que el sistema puede adoptar. La fortaleza de esta técnica radica en la posibilidad de modelar problemas complejos de una manera no cuantitativa (Pidd, 1996). En consecuencia, el AM es por su naturaleza un método para estructurar problemas más que para resolverlos.

El análisis morfológico es una matriz que engloba todas las posibles combinaciones teóricamente concebibles de los enfoques y configuraciones tecnológicas. Este análisis identifica la tecnología conocida y predice la tecnología futura para explayar posibilidades que todavía no son usadas o exploradas.

El análisis morfológico de patentes se presta muy bien para:

- $\quad$ Conocer el estado del arte y, no comenzar de cero ni inventar el hilo negro

- $\quad$ Analizar patentes de la competencia que nos pudieran causar problemas para desarrollar nuestros propios productos. Moverse en el mundo de las patentes es similar a aprender a moverse en una gran ciudad donde abundan grandes y pequeñas avenidas bloqueadas porque son propiedad de alguien.

- Descubrir huecos de oportunidad tecnológica

Esta técnica se utiliza no sólo para identificar las posibles respuestas a un problema o para visualizar significados de un efecto tecnológico, sino que más bien constituye un sentido de creatividad ya que se identifican nuevos sistemas y conceptos a través de nuevas combinaciones en la matriz. Por lo que es utilizada para pronosticar nuevos sistemas tecnológicos posibles. Destacando que la matriz es meramente un enfoque cualitativo o descriptivo.

El primer paso para su construcción es la realización de un riguroso análisis para la identificación de los componentes de un sistema dado. Estos componentes pueden describir un producto o un proceso complejo.

Después, los componentes son identificadas como A, B, C, etc., y son puestos en la primera columna vertical de la

ISSN: 07 I8-2724. (http://www.jotmi.org) 
matriz. En la segunda columna vertical son anotados los significados empleados en la operación del sistema. La segunda columna vertical es encabezada como "I", por lo que la descripción del sistema conocido puede ser designada como $\mathrm{Al}, \mathrm{BI}, \mathrm{Cl}$, etc.

\section{Análisis de patentes (AP) y minería de texto}

Recientemente, la minería de texto, que es usada para extraer información valiosa de volúmenes grandes de texto, ha sido adoptada para explorar relaciones complejas en documentos patentes. La minería de textos (text mining) es una metodología cuyo propósito consiste en extraer información relevante y a veces desconocida de manera automática dentro de grandes volúmenes de información textual, normalmente en lenguaje natural y por lo general no estructurada (Brun y Senso, 2004).

Por su parte, el análisis de patentes ayuda a descubrir las tendencias cambiantes en el énfasis de I+D y a pronosticar el futuro mediante la recuperación y relación de documentos técnicos (Porter, 2009). Varios estudios sobre la extracción y análisis de texto de patentes sugieren el proceso de extraer documentos de bases de datos para el análisis de oportunidades tecnológicas o procesos automatizados para obtener el conocimiento provechoso de textos (Zhu et al. 1999). La oportunidad y la utilidad de minería de texto son materializadas en el mapa de patentes (Yoon, et al. 2002) y el árbol de patentes (Yoon y Park, 2004a) en el cual puede visualizar la relación entre ellas de una manera gráfica.Además, la aplicabilidad de la minería de texto en el análisis de patentes es demostrada comparando el resultado de este método con él de análisis de citas de patentes (Yoon y Park, 2004b).

Cavallucci, Rousselot y Zanni (20I I) resumen las principales contribuciones y cuellos de botella de en la investigación mediante el uso de patentes en el ámbito de las prácticas relacionadas con TRIZ [Lenguajes inaccesible o patentes mal escaneados, necesidad multidisciplinaria en la cobertura de determinados dominios, diferentes sistemas conceptuales de diferentes expertos y experiencia en la gestión de contradicciones]. Además, proponen un método de diseño de invención el cual consiste en un marco con el objetivo de extraer y representar los conocimientos de los expertos y llenar una ontología ya construida de diseño inventivo. En una patente, algunos datos necesarios para rellenar la Ontología de Diseño Inventiva (Zanni, Cavallucci \& Rousselot, 2009) están ocultos o el conocimiento está disperso en el texto. La figura en el anexo I nos muestra cómo las patentes pueden poblar esta ontología. El diagrama de flujo muestra dos procesos paralelos de fuente de conocimiento. Con el fin de no interferir entre ambos y evitar redundancias en los elementos recogidos, se expone un proceso en el diagrama de flujo el cual consiste en pedir a un experto en una etapa específica de la construcción del modelo validar o eliminar lo que es producido por la minería de patentes. Gracias a la naturaleza organizada de las patentes, es posible utilizar la estructura para buscar un conocimiento específico en una sección específica.

\begin{tabular}{|c|c|c|c|c|}
\hline $\begin{array}{l}\text { Parámetros } 0 \\
\text { dimensiones }\end{array}$ & \multicolumn{4}{|c|}{$\begin{array}{c}\text { Características } \\
\text { (de las soluciones posibles, se muestran dos) }\end{array}$} \\
\hline Agua & $\begin{array}{l}\text { - Elemento de } \\
\text { calentamiento } \\
\text { (interno) }\end{array}$ & \multicolumn{2}{|c|}{ Flama abierta } & 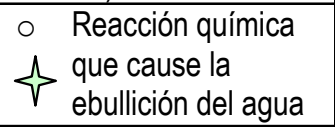 \\
\hline Añadir café & $\begin{array}{l}\text { Mediante cucharadas a } \\
\text { mano }\end{array}$ & \multicolumn{2}{|c|}{$\circ \begin{array}{l}\text { Con una copa a l } \\
\text { medida }\end{array}$} & - Elemento medidgr \\
\hline Filtrar café & - Papel filtro $\widehat{\sim}$ & \multicolumn{2}{|c|}{ o Cerámica porosał } & \begin{tabular}{|l|} 
Centrifuga \\
\end{tabular} \\
\hline Mantenerlo caliente & $\begin{array}{l}\text { Material térmico } \\
\text { aislante }\end{array}$ & \multicolumn{2}{|c|}{ - $\begin{array}{l}\text { Calentamiento } \\
\text { continuo }\end{array}$} & $\circ \begin{array}{ll}\text { Unidad de } \\
\text { calentamiento }\end{array}$ \\
\hline Servirlo & $\circ$ Válvula $\&$ & \multicolumn{2}{|l|}{ Bomba } & - $\begin{array}{l}\text { Verter sobre dos } \\
\text { recipientes }\end{array}$ \\
\hline $\begin{array}{l}\text { Solución } \\
\text { (ejemplos) }\end{array}$ & \multicolumn{2}{|c|}{$\begin{array}{l}\text { Sol } 1 \\
\text { Infusión = reacción química } \\
\text { Añadir café = con una copa } \\
\text { Filtrado = cerámica } \\
\text { Mantenerlo caliente = unidad de } \\
\text { Servir = válvula } \quad \text { calentamiento }\end{array}$} & \multicolumn{2}{|c|}{$\begin{array}{l}\bullet \quad \text { Sol } 2 \\
\text { Infusión = elemento de calentamiento } \\
\text { Añadir café = elemento medidor } \\
\text { Filtrado = papel filtro } \\
\text { Mantenerlo caliente = añadir calor } \\
\text { Servir = dos recipientes }\end{array}$} \\
\hline
\end{tabular}

Tabla I. Ejemplo de una matriz morfológica: Formas y Atributos. Fuente: Kohn y Niethammer (2004)

ISSN: 07 I8-2724. (http://www.jotmi.org)

Journal of Technology Management \& Innovation (c) Universidad Alberto Hurtado, Facultad de Economía y Negocios. 


\section{Análisis morfológico (AM) con base en palabras clave}

Para esta investigación se propone un AM basado en palabras clave. El AM, es un método no cuantitativo que conduce a la invención estructurada, es un análisis sistemático de la estructura actual y futura de tecnología y un estímulo fuerte para la invención de nuevas alternativas (Ritchey, 2009). Aunque con frecuencia es presentado en numerosos documentos y manuales, el empleo práctico del AM rara vez es llevado a cabo en las empresas de América Latina.

El fenómeno es atribuible a tres motivos: i) no hay ningún modo científico o sistemático de establecer las dimensiones y formas (Wissema, 1976). ii) El AM es típicamente un método cualitativo que depende de la opinión de los expertos. Generalmente es utilizado sólo para la planificación a corto plazo en un tiempo específico, llenando huecos entre la tecnología actual y la tecnología emergente. Finalmente, las alternativas son difíciles de priorizar con el AM tradicional. iii) De tal manera que, la lista de configuraciones de tecnología posibles siguen acumulándose, pudiendo llegando a confundir a investigadores en la identificación de brechas tecnológicas.

El objetivo principal de este trabajo es utilizar el AM basado en palabras clave propuesto por Yoon y Park (2005) el cual es apoyada por un procedimiento sistemático para obtener la morfología de tecnologías.

Con el objetivo potenciar el AM se propone la utilización de palabras clave extraídas de documentos de patentes. Lo cual puede añadir un rasgo cuantitativo al proceso de pronóstico tecnológico por medio del análisis de datos de patentes y así mejorar su poder explicativo relacionando tecnologías substitutas $O$ análogas. Varios estudios sobre el descubrimiento de conocimiento han asumido que un conjunto de palabras clave de un documento representa el tema principal del mismo (Zhu et al. 1999; Feldman, 1998).

Por lo tanto, es posible identificar todas las configuraciones de la tecnología utilizadas trazando un mapa de las palabras clave en las patentes existentes en una morfología determinada. La Tabla I ejemplifica la matriz morfológica.

Esta matriz tiene cinco dimensiones, que respectivamente están descompuestas en tres formas, por lo tanto hay 35 = 243 combinaciones posibles, en la última fila se muestran dos de ellas quedando 24I por ejemplificar. En su momento, para nuestro caso, serán mostradas las combinaciones protegidas en tres patentes (Ver Tabla 5).

El procedimiento de AM para emprender el análisis de oportunidades de tecnología está compuesto de cinco pasos:
I) Recopilación de documentos de patente del área tecnológica de interés en la página de United States Patent and Trademark Office (USPTO http://www.uspto.gov) y de Espacenet www.espacenet.com).

2) Con base en los títulos y abstract de las patentes el experto definió las dimensiones y las formas de las tecnologías disponibles para generar una matriz morfológica.

3) Revisión de la matriz para extraer las palabras clave de los documentos de patentes por medio de minería de texto, (en nuestro caso utilizamos el Software InsightPro).

4) Revisión con el experto de la morfología de las patentes existentes con base en las palabras clave.

5) Identificación de las configuraciones llenas (tecnologías existentes) y las vacías (aún no desarrolladas). De todas las alternativas resultantes posibles por medio de la eliminación de las configuraciones existentes.

\section{Estudio de Caso: Desarrollo de un Producto de Seguridad Vehicular}

A continuación presentamos los avances del caso referido a la investigación Universidad - Empresa, relacionado con el desarrollo de dispositivos electrónicos para la seguridad de vehículos semicompactos para los mercados emergentes (Brasil, México y Argentina).

\section{Oportunidad y necesidades del mercado vehicular}

El posicionamiento de la industria automotriz mexicana ha sido el motor del crecimiento del comercio exterior del país. México es el $9^{\circ}$ productor mundial y el 3er exportador al mercado de Estados Unidos. Es por tanto una industria estratégica que amerita una planeación integral desde el punto de vista del país y un mayor involucramiento de las universidades. Una muestra de los indicadores de oportunidad (necesidades) de mercado son los siguientes:

- Las ventas mundiales de vehículos podrían alcanzar más de 370 millones de unidades en 2013 y 715 millones de unidades en 2018, según un estudio de la firma de consultoría Booz \& Company, que apunta que el momento de mayor crecimiento para el sector de la automoción está por llegar. Sin embargo, este "estallido" del mercado se producirá fundamentalmente por razones sociodemográficas en economías emergentes.

- Entre los cambios tecnológicos importantes del sector está la transformación de sistemas mecánicos en electrónicos (como en la industria aeronáutica): sistemas de dirección y frenos entre otros controles. Gobierno del Estado de Chihuahua (2004).

- $\quad$ El Reglamento (CE) $\mathrm{n}^{\circ}$ 78/2009 del Parlamento Europeo y del Consejo (2009), de I4 de enero de 2009 endurece los requisitos técnicos de seguridad de automóviles y vehículos comerciales ligeros, con el objetivo de reducir

ISSN: 07 I8-2724. (http://www.jotmi.org)

Journal of Technology Management \& Innovation (c) Universidad Alberto Hurtado, Facultad de Economía y Negocios. 
los daños a peatones y ciclistas en caso de atropello. La Eurocámara recordó, en un comunicado, que cada año 8.000 peatones y ciclistas pierden la vida y 300.000 resultan heridos en accidentes en las carreteras de la UE-25 (sin Bulgaria y Rumanía).

- $\quad$ En cuanto al tipo de colisión, se observa que en la Argentina (Donnet, 2007), la colisión frontal es la que más se repite en los siniestros con el $29 \%$ del total, seguida por el vuelco con el $16 \%$ y la colisión trasera con el $16 \%$, valores similares a los que se dan internacionalmente.

- Los automóviles del futuro serán respetuosos con el medio ambiente, tomarán sus propias decisiones para evitar los accidentes y adaptarán su diseño a cada estilo de vida, pero su mayor eficiencia y seguridad reducirán la libertad de acción del conductor. (Gómez, 2005).

- El problema de los accidentes viales es multifactorial; se detectan las siguientes causas como las principales: (a) el poco respeto al reglamento de vialidad (no guardar la distancia de seguridad entre vehículos, virar indebidamente, no respetar señalamientos viales, no respetar semáforo, invadir carril contrario y cruce imprudencial de peatones/ciclistas/ mascotas) (b) por reflejos disminuidos en conductores (por manejar cansados, por usar el teléfono celular mientras conducen o por ser conductores ancianos), (c) conductores sin suficiente pericia para conducir (d) conductas agresivas, (e) factores climáticos (lluvia y/o niebla), (f) señalización vial insuficiente/deficiente, y $(g)$ presencia de objetos varios $y$ baches en las vialidades.

Específicamente hablando del mercado mexicano de los automóviles podemos decir que:

- El equipamiento de los autos incluyendo dispositivos de seguridad, de asistencia al conductor en el manejo del vehículo, de entretenimiento y de comunicación, es proporcional al costo de los autos, siendo los de más bajo costo los menos equipados y los autos de lujo, también llamados "Autos Inteligentes" los mejor equipados. En otras palabras, el auto más caro es el auto más seguro. El precio de los autos bien equipados, los llamados "autos inteligentes" que incluyen algunos sistemas de advertencia de accidentes viales, oscila entre $\$ 25,000$ dlls. y $\$ 40,000$ dlls., lo que los hace poco accesibles para el grueso del mercado automotriz mexicano.

- $\quad$ De acuerdo con el INEGI, el $28.32 \%$ de los autos vendidos en México en 2009 y el $27.86 \%$ en 2010 son subcompactos (INEGI, 20II).

- De acuerdo con información de la base de datos "Inteligencia de Mercados" (IM_ITESO 2007), la cantidad de autos nuevos que se compran en México ha ido siempre de la mano de la salud de la economía nacional. No obstante, datos consultados en el INEGI (INEGI 20I2) muestran la siguiente tendencia a lo largo de 3 décadas: los autos más comprados en México son en primer lugar del tipo compac- to (por ejemplo: Jetta, Tiida, Bora, etc.), en segundo lugar los de tipo subcompacto (por ejemplo:Tsuru, Chevy, Aveo, etc.) y en tercer lugar los de uso múltiple (por ejemplo: Journey, CR-V, Escape, etc.) y solo un pequeño porcentaje de autos del tipo deportivo y de lujo. Es importante señalar que, el sector más amplio del mercado automotriz mexicano es el de los autos compactos.

- $\quad$ En general las grandes armadoras instaladas en el país (GM, Ford, Chrysler,Volkswagen, Honda, Nissan, Peugeot, Renault, etc.) incorporan las innovaciones tecnológicas de equipamiento de los autos provenientes de sus filiales en EU, Asia o Europa, no obstante que, el equipamiento de los autos de lujo varía de país a país. En México, identificamos a dos empresas, Continental y Delphi realizando desarrollos industriales para aplicaciones de electrónica automotriz. También se identificó al ITESM con el Cima (Centro de Investigación en Ingeniería Automotriz) y el Cediam (Centro de Desarrollo para la Industria Automotriz Mexicana) realizando proyectos de manufactura automotriz. Sin embargo, a la fecha de elaboración de esta propuesta no se detectaron instituciones educativas mexicanas que trabajen sobre dispositivos auxiliares de seguridad en automóviles.

- Es importante señalar que, de acuerdo con datos de la Dirección de Investigación Vial de la Dirección General de Seguridad Vial del Estado de Jalisco (SVT Jalisco 20I0), tan solo en la zona metropolitana de Guadalajara ocurren alrededor de 51,000 accidentes viales por año sin presencia de alcohol, de los cuales, el $89 \%$ corresponde a choques por alcance entre vehículos particulares seguido del choque de vehículos contra objetos fijos (8.2\%). En el anexo II se desglosan zonas y cruceros de mayor accidente y causas aparentes de ellos para el caso local de Guadalajara, mismos que podrían considerarse en el diseño de la solución.

\section{Análisis morfológico de patentes de "anticolisiones"}

El conocimiento tecnológico se desarrolla siguiendo el modelo de análisis morfológico. Los pasos que se siguieron en su desarrollo fueron:

\section{Paso I. Recopilación de documentos de patente.}

El primer paso de la metodología de AM basado en palabras clave es la búsqueda y recopilación de los documentos de patente del área tecnológica de interés en la página de USPTO y ESPACENET.

Para el caso de estudio se limitó la selección de años desde 1976 a la fecha. El principal criterio utilizado fue "anticollision and (vehicle or communication or automobile)" en los campos de "Claim" (reivindicación) o "Title" (título). En una segunda vuelta se añadieron otros criterios señalados por el experto en virtud de haber encontrado nuevas palabras 
clave de interés por ejemplo "infrared (IR) sensor or videocamera". El resultado acumulado de la consulta fue de 604 patentes.

\section{Paso 2: Extracción de las palabras y construcción del diccionario tecnológico.}

El proceso del desarrollo del diccionario de tecnología está compuesto de varios pasos: Las palabras clave fueron extraídas de las patentes por medio de la minería de texto utilizando el sistema de software "InsightPro". Después de la eliminación de las palabras suplementarias (no relacionadas con la tecnología) se registró la frecuencia de dichas palabras por el mismo sistema.Ver Tabla 2.

\section{Paso 3: Definición de la estructura morfológica.}

Se llevó a cabo una reunión con el experto para analizar las características de las 604 patentes resultantes de la búsqueda y proceder a crear una matriz morfológica la cual muestra las dimensiones (encabezados de la Tabla) y formas (filas de la Tabla) más importantes de la tecnología en estudio. Dicha matriz se tomó como base para desarrollar otros pasos de la metodología.Ver Tabla 3.

Es importante mencionar que esta matriz morfológica es resultado de la experiencia y conocimiento del experto en combinación con el método propuesto.

\section{Paso 4: Representación de las patentes recopiladas.}

Los vectores por palabra construidos y representados en la Tabla 3 se relacionan con las dimensiones y formas previa-

\begin{tabular}{|c|c|c|c|c|}
\hline \multirow{6}{*}{$\begin{array}{l}\text { Select Text Section } \square \\
\Gamma \text { Title } \\
\varpi \text { Abstract } \\
\nwarrow \text { Independent Claims } \\
\varpi \text { Claims }\end{array}$} & \multicolumn{4}{|c|}{-Keywords } \\
\hline & \multicolumn{2}{|r|}{ Keywords } & \multicolumn{2}{|r|}{$\#$} \\
\hline & \multicolumn{2}{|r|}{$A$} & \multicolumn{2}{|r|}{$=$} \\
\hline & $\omega_{2}$ & SAMS & $\mathrm{X}$ & 30 \\
\hline & 舴. & Samplifiers & $\mathrm{X}$ & 72 \\
\hline & (⿻)丨 & Sarray antennaS & $\mathrm{x}$ & 2 \\
\hline \multirow{5}{*}{ Generate Keywords $\downarrow$} & 幽 & Saudio signal\$ & $\mathrm{x}$ & 6 \\
\hline & (⿻)丨子巾 & Scircuits & $\mathrm{K}$ & 280 \\
\hline & ex. & Sdemodulator' & $\mathrm{x}$ & 26 \\
\hline & $\omega_{2}$ & Sdifferentials & $\mathrm{x}$ & 30 \\
\hline & $\omega_{2}$ & SDigitalSvideoScameraS & $\mathrm{x}$ & 2 \\
\hline \multirow{2}{*}{ Clear Keywords } & $\omega_{2}$ & SElectric signals & $\mathrm{X}$ & 5 \\
\hline & $\omega_{2}$ & Sfilters S & $\mathrm{x}$ & 46 \\
\hline \multirow{4}{*}{$\begin{array}{l}\text { Remove Keywords } \\
\text { Count Cut Off } 1 \doteq\end{array}$} & Ata & Simages & $\mathrm{K}$ & 76 \\
\hline & 幽 & SIRS & $\mathrm{X}$ & 27 \\
\hline & $\omega_{2}$ & Slasers\$ & $\mathrm{X}$ & 7 \\
\hline & As. & SLIDARS & $\mathrm{x}$ & 3 \\
\hline \multirow[t]{2}{*}{ Remove Keywords } & ex & SMechanical signals & $\mathrm{x}$ & 1 \\
\hline & $\omega_{x}$ & SMicrowaves & $\mathrm{x}$ & 57 \\
\hline \multirow{4}{*}{$\begin{array}{l}\text { Do you want to } \\
\text { Automatically Merge } \\
\text { similar Keywords? }\end{array}$} & $\omega_{2}$ & SRadarS & $\mathrm{x}$ & 86 \\
\hline & $\omega_{2}$ & Srectifiers\$ & $\mathrm{x}$ & 1 \\
\hline & 10 & SUltrasonicS & $\mathrm{x}$ & 32 \\
\hline & 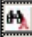 & SvideocameraS & $\mathrm{x}$ & 1 \\
\hline $\begin{array}{l}\text { Import Keywords from } \\
\text { Excel }\end{array}$ & 皮 & Swireless signal\$ & $\mathrm{x}$ & 8 \\
\hline
\end{tabular}

Tabla 2. Extracción y Frecuencia de Palabras Clave por Patente. mente definidas en la Tabla 4 con objeto de representar el mapa de las tecnologías existentes en cada patente.

De la Tabla 4 el primer análisis consistió en determinar la existencia de combinaciones posibles por tipo de sensor (de la columna 2 ), de tal manera que se dichas combinaciones son las siguientes: $(5 \times 5 \times 2 \times 5 \times 3 \times 3)=2,250$. Así mismo se pueden observar las morfologías de las tecnologías protegidas en relación a la función (columna I), y tipo de sensor utilizado (columna 2). El resultado de este análisis se muestra en la Tabla 5 para mostrar la morfología de las tres patentes con mayor interés $y$, al hacerlo para todas las patentes, se podrá determinar el estado del arte.

\section{Paso 5 Análisis morfológico.}

El AM producirá varias combinaciones morfológicas no desarrolladas. Por lo tanto, se requiere determinar la prioridad del potencial de la nueva tecnología para reducir el conjunto de oportunidades a analizar. La extensión de la morfología se logra por dos métodos-la diferenciación y la diversificación. Donde la diferenciación es aplicable a las funciones $(A, B, C, D, E, F)$. Por su parte la diversificación ocurre cuando se agrega un nuevo sensor a la matriz generando así una nueva característica del producto que la vez deberá tener formas para definir esta nueva característica $(1,2,3,4$..). Ver Figura 3. Este trabajo requiere del conocimiento y creativi-

\begin{tabular}{|c|c|}
\hline No. de Patente & Morfologia de la patente \\
\hline US20020003571 & A1 $\rightarrow 5-$ B1,2,4,5 - E1 $\rightarrow 3$ \\
\hline US20050150740 & A2,4,5 - B2,4,5 - D1,2,3,4 - F1 \\
\hline US7849993 & A4,5 - B2,4,5 - D1 $\rightarrow 4$ \\
\hline
\end{tabular}

Tabla 5. Matriz Morfológica

\begin{tabular}{|c|c|}
\hline Función & Tipo de sensor usado \\
\hline \multirow[t]{6}{*}{$\begin{array}{l}\text { Detectar objetos y determinar la velocidad } \\
\text { relativa }\end{array}$} & $\begin{array}{l}\text { Microwave radar (an array of two antennas having } \\
\text { two overlapping radiation patterns) }\end{array}$ \\
\hline & $\begin{array}{l}\text { LIDAR (an array of InfraRed lasers } \\
\text { with non-overlapping fields of view) }\end{array}$ \\
\hline & Digital video cameras \\
\hline & \begin{tabular}{|l} 
Infrared (IR) sensors or videocamera. \\
.
\end{tabular} \\
\hline & Ultrasonic sensors . \\
\hline & ¿Qué usa para evaluar señales? \\
\hline \multirow{3}{*}{ Acción 1: Evalúa señales recibidas } & Microwave radar \\
\hline & $\begin{array}{l}\text { AM circuit (demodulator, rectifiers, filters, } \\
\text { differential amplifier and adjustable threshol } \\
\text { circuit) }\end{array}$ \\
\hline & ¿En qué formato está la señal enviada? \\
\hline \multirow[t]{5}{*}{ Acción 2: Genera señal para el propio auto } & Electric signal \\
\hline & Optic signal \\
\hline & Mechanical signal \\
\hline & Image \\
\hline & Audio signal \\
\hline \multirow[t]{2}{*}{ Acción 3: Envío de señal a otros autos } & Wireless signal \\
\hline & Optic signal \\
\hline \multirow{3}{*}{$\begin{array}{l}\text { Acción 4: Recepción de señal proveniente de otro } \\
\text { auto. }\end{array}$} & Wireless signal \\
\hline & Optic signal \\
\hline & Audio signal \\
\hline
\end{tabular}

Tabla 3. Matriz de estructura morfológica propuesta por el experto.

ISSN: 07 I8-2724. (http://www.jotmi.org)

Journal of Technology Management \& Innovation (c) Universidad Alberto Hurtado, Facultad de Economía y Negocios. 
dad del experto tecnológico quien, en nuestro caso, encontró una oportunidad de desarrollo.

\section{Conclusiones}

La idea principal plantea al AM como una herramienta de detección de oportunidades tecnológicas, la cual sería una forma de comenzar con el proceso de invención. En términos de innovación requiere complementarse con la detección de las necesidades de los clientes para relacionarlas y definir una idea de innovación, ya que de lo contrario se estaría produciendo una patente sin aplicación para el mercado.

A partir de la construcción de la matriz de la estructura morfológica (Tabla 5) y del análisis de los títulos y abstracts de las primeras patentes se realizó el primer hallazgo: el experto descubrió algunas dimensiones que antes no había considerado en su estructura mental y a la vez se pudo hacer una búsqueda más extensa de otras patentes en estas nuevas dimensiones.

La generación de un diccionario tecnológico permitió desarrollar un mapa morfológico de las tecnologías protegidas y detectar las oportunidades de tecnologías por desarrollar con posibilidades de propiedad industrial.

Se propone el análisis morfológico basado en palabras clave como una herramienta de prospectiva tecnológica cuyo objetivo es facilitar al experto el proceso inventivo a partir de lo ya creado pero permitiendo, con base en sus conocimientos, identificar huecos tecnológicos en las patentes que se toman como base de estudio y resolverlos creativamente y con posibilidades de factibilidad económica, lo cual deberá ser corroborado por el equipo de desarrollo.

Además, el proceder de esta forma permite iniciar a partir del estado del arte para no inventar "el hilo negó", lo cual ahorra tiempo y disminuye costos en el desarrollo de nuevos productos, y es básico para la gestión de la propiedad industrial.
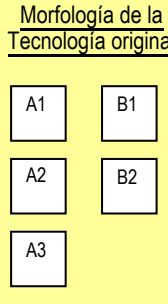$$
\text { (1) }
$$

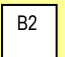

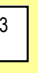
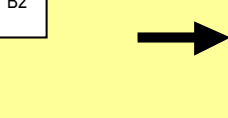

Figura 3. Extensión de formas y dimensiones mediante diferenciación y diversificación. Fuente:Yoon and Park (2005)

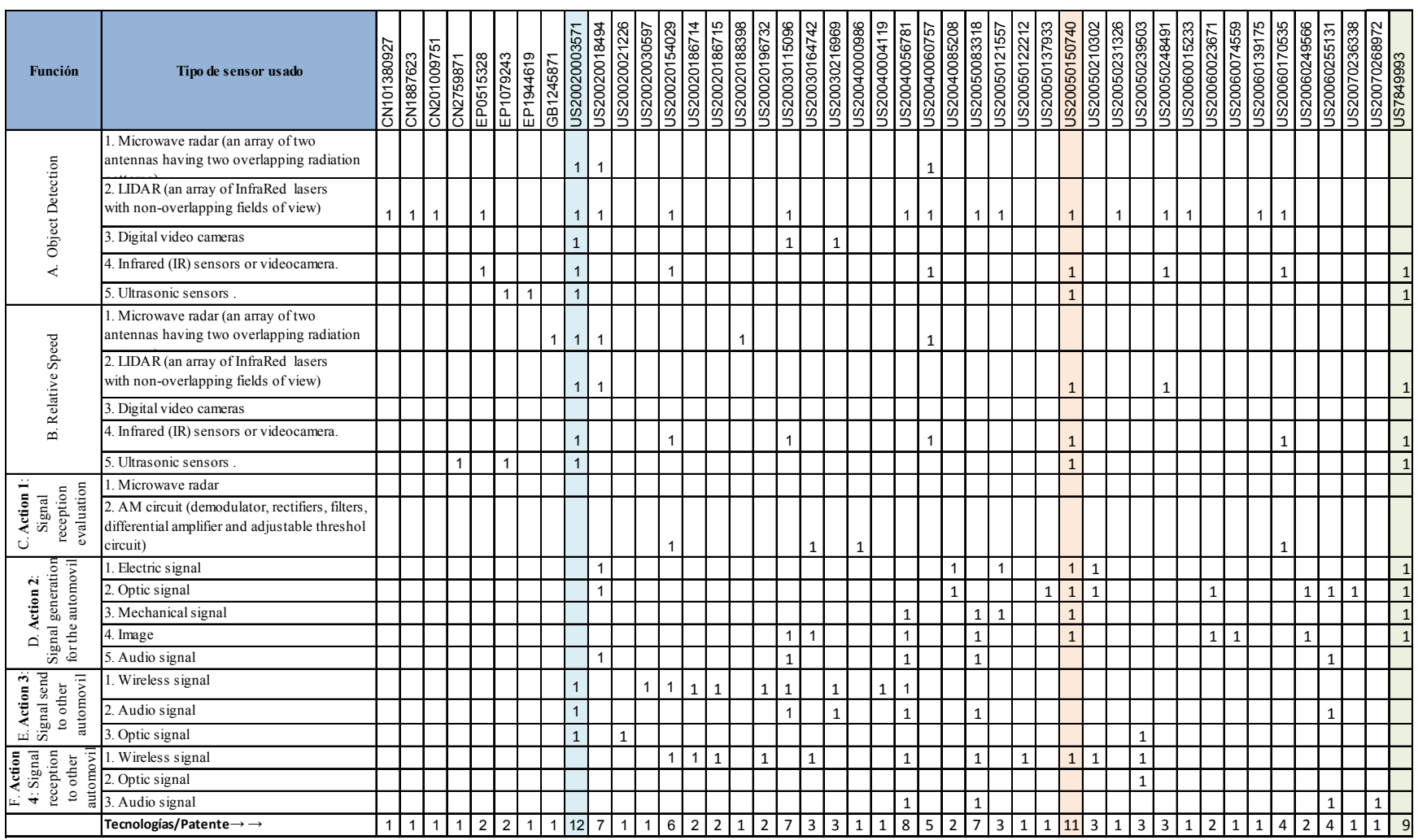

Tabla 4. Representación de Patentes Recopiladas

ISSN: 07I 8-2724. (http://www.jotmi.org)

Journal of Technology Management \& Innovation (c) Universidad Alberto Hurtado, Facultad de Economía y Negocios. 


\section{Referencias}

BRUN R. E., Senso J.A. (2004) Minería textual. El profesional de la información, I3(I), II-27.

CAVALLUCCI, D., Rousselot, F., Zanni, C. (20II) Using patents to populate an inventive design ontology. Procedia Engineering (9), 52-62.

DONNET E. y Filgueira E. Filgueira (2007) Vehículos de transporte de pasajeros de Doble Piso. Estudio técnico: evaluación de las condiciones de seguridad. Ed. edUTecNe, Buenos Aires - Argentina.

FELDMAN, R., Fresko, M., Hirsh, H.; Aumann, Y., Liphstat, O., Schler, Y., Rajman, Y. (1998). Knowledge management: a text mining approach. 2da. Conferencia Internacional: Practical Aspects of Knowledge Management. Basilea, Suiza.

GOBIERNO del Estado de Chihuahua (2004). Industria Automotriz y de Autopartes. Estrategia para la Integración de su Agrupamiento Industrial. http://www.contactopyme.gob. $\mathrm{mx} /$ estudios/docs/automotriz_autopartes_chihuahua.pps.

GÓMEZ, M. (2005). El Coche del Futuro. El País, domingo 5 de junio. Visto el 18 de abril de 2005 en http://elpais.com/ diario/2005/06/05/eps/ I I 79528I3_8502 I5.html

INEGI, (20I2). La industria automotriz en México 20I2. Serie estadísticas sectoriales. Instituto Nacional de Estadística y Geografía No. 27. Aguacalientes, México.

KOHN, S., Niethammer, R.. (2004). Why patent data can be a good source of comparative technology intelligence in new product development, Visions Magazine, January, VIII(I), I6-I9.

MYERS, S., Marquis D. G., (1969). Successful industrial innovation, Washington D.C., National Science Foundation.

PARLAMENTO Europeo Y Del Consejo (2009). Reglamento (CE) $n^{\circ} 78 / 2009$ Visto el 18 de abril de 2013 en http:// europa.eu/legislation_summaries/internal_market/single_ market_for_goods/motor_vehicles/technical_implications_ road_safety/mi0047_es.htm.

PEDROZA,A., Ortiz, S. (2008). Gestión estratégica de la tecnología en el predesarrollo de nuevos productos. Journal of Technology Management \& Innovation, 3 (3), I I2-I 22.

PIDD, M. (1996). Tools for thinking-modeling in management science, Wiley, London,
PORTER, A. (2009). Text mining for technology forecasting. En: J.C. Glenn, T.J. Gordon (Eds.) Futures Research Methodology Version 3.0 [CD-ROM]. Chap. 3. The Millennium Project, (DC, United States).

RITCHEY, T. (2009). Morphological Analysis. En Glenn, J.C; Gordon, T. J. (Eds.) Futures research methodology, Version 3.0 [CD-ROM]. Chap. 17.The Millennium Project, DC, United States.

SECRETARIA de Vialidad y Transporte del Estado de Jalisco. Información entregada en mano.

WISSEMA, J.G. (1976). Morphological analysis: its application to a company TF investigation, Futures 8(2), I46-153.

YOON, B.U., Yoon, C.B.; Park, Y.T. (2002). On the development and application of a self-organizing feature map-based patent map. R\&D Management. 34(4), 29I-300.

YOON, B.U., Park, Y.T. (2004a). A text-mining-based patent network: analytical tool for high-technology trend. J. High Technoly Management Research. 15, 37-50.

YOON, B.U., Park,Y.T. (2004b). Evaluating the applicability of text mining-based patent analysis. Conferencia de Ingeniería Industrial Proc. 33rd Int. Corea del Sur, Jeju.

YOON, B.U., Park, Y.T. (2005). A systematic approach for identifying technology opportunities: keyword-based morphology analysis. Technological Forecasting \& Social Change 72(2), I45-160.

ZANNI, C., Cavallucci, D., Rousselot, F. (2009). An ontological basis for computer aided innovation. Computers in Industry, 60, October, 563-574.

ZHU, D., Porter, A.L. (2002). Automated extraction and visualization of information for technological intelligence and forecasting Technology. Technological Forecasting \& Social Change. 69(5), 495-506. 


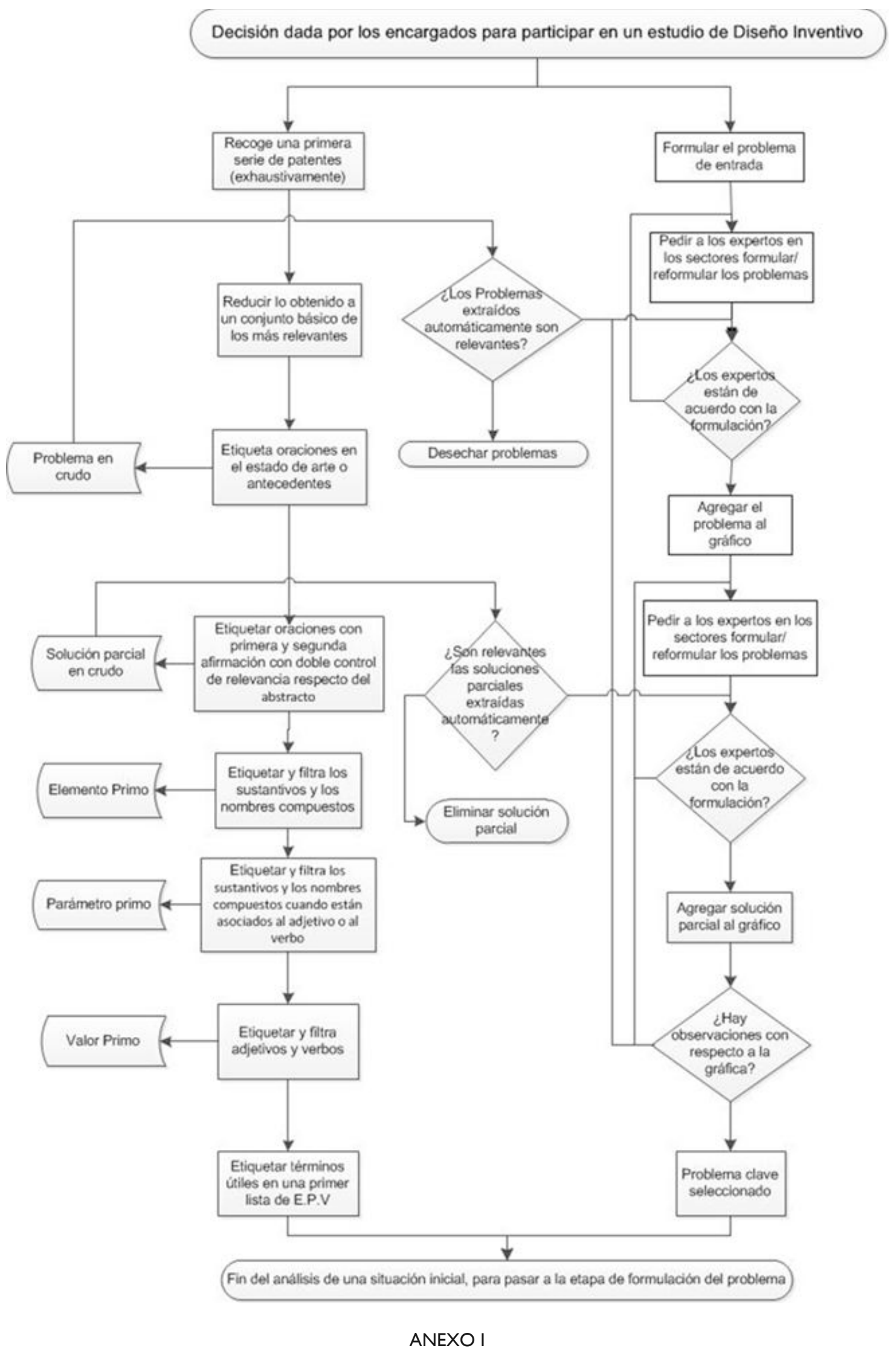

Fuente: Cavallucci, Rousselot \& Zanni (20II)

ISSN: 07I8-2724. (http://www.jotmi.org)

Journal of Technology Management \& Innovation (c) Universidad Alberto Hurtado, Facultad de Economía y Negocios. 


\section{ANEXO $\|$}

La arteria con mayor índice de accidentes es el Periférico desde el 2005, independientemente de su cruce, de acuerdo a las estadísticas de la Dirección de Investigación Vial de la Dirección General de Seguridad Vial, en el 2009 las zonas o cruceros con mayor incidencia de accidentes viales se pueden ver en la Tabla $A$.

Entre las causas aparentes de los accidentes podemos encontrar las que se anotan en la Tabla B, estas causas no se tienen cuantificadas.

\begin{tabular}{|l|l|}
\hline Cruceros & Accidentes \\
\hline Periférico - Av. López Mateos & 531 \\
\hline Periférico - José Parres Arias & 299 \\
\hline Periférico - Av. Colón & 297 \\
\hline Periférico - 5 De Mayo Ocotán & 253 \\
\hline Av López Mateos - Av. Mariano Otero & 246 \\
\hline Periférico - Juan De La Barrera & 242 \\
\hline Periférico - Iteso & 224 \\
\hline Periférico - Morelos (San Sebastianito) & 215 \\
\hline Calz Lázaro Cárdenas - Av Solidaridad Iberoamericana & 208 \\
\hline Periférico - Calz Federalismo & 195 \\
\hline Periférico - Av. Alcalde & 187 \\
\hline Calz. Lázaro Cárdenas - 8 De Julio & 183 \\
\hline Periférico - Av. Tabachines & 181 \\
\hline Calz. Lázaro Cárdenas - Av Colón & 178 \\
\hline Periférico - Av. Vallarta & 173 \\
\hline Periférico - Av. Guadalupe & 163 \\
\hline Periférico - Av. Mariano Otero & 158 \\
\hline Periférico - Av. Acueducto Zap & 156 \\
\hline Av Solidaridad Iberoamericana - Plan De Ayala & 156 \\
\hline Periférico - 8 De Julio & 155 \\
\hline
\end{tabular}

Tabla A. Zonas o Cruceros con mayor Incidencia de Accidentes viales en la ZMG [Zona Metropolitana de Guadalajara incluye los municipios de Guadalajara, Zapopan, Tlaquepaque y Tonalá] en 2009

Fuente: Dirección de Investigación Vial de la Secretaría de Vialidad y Transporte del Estado de Jalisco

\begin{tabular}{|l|}
\hline Causas aparentes \\
\hline Virar indebidamente \\
\hline No guardar distancia de seguridad \\
\hline No respetar señalamientos \\
\hline No respetar alto de semáforo \\
\hline Cruzar imprudencialmente (peatón) \\
\hline Invadir carril contrario \\
\hline
\end{tabular}

Tabla B. Causas aparentes de los accidentes viales entre particulares en 2009 en la ZMG

Fuente: Dirección de Investigación Vial de la Secretaría de Vialidad y Transporte del Estado de Jalisco

ISSN: 07 I8-2724. (http://www.jotmi.org)

Journal of Technology Management \& Innovation (C) Universidad Alberto Hurtado, Facultad de Economía y Negocios. 